\title{
Morfología del cariotipo de Luma apiculata (DC.) Burret (Myrtaceae)
}

\section{Karyotype morphology of Luma apiculata (DC.) Burret (Myrtaceae)}

\author{
Pedro Jara-Seguel ${ }^{1 *}$, Paz Cárcamo-Fincheira ${ }^{1}$, Claudio Palma-Rojas ${ }^{2} \&$ Elisabeth von Brand ${ }^{3}$ \\ ${ }^{1}$ Escuela de Ciencias Ambientales, Facultad de Recursos Naturales, Universidad Católica de Temuco, Casilla 15-D, Temuco- \\ Chile. \\ ${ }^{2}$ Departamento de Biología, Facultad de Ciencias, Universidad de La Serena, Casilla 599, La Serena-Chile. \\ ${ }^{3}$ Departamento de Biología Marina, Facultad de Ciencias del Mar, Universidad Católica del Norte, Casilla 117, Coquimbo, \\ Chile. \\ *pjara@uct.cl
}

\begin{abstract}
Luma apiculata (DC.) Burret, had a symmetric karyotype, with $2 \mathrm{n}=22$ small sized chromosomes, ranging between 2.2 $\mu \mathrm{m}$ and $0.8 \mu \mathrm{m}$. The haploid karyotype formula was $9 \mathrm{~m}+2 \mathrm{sm}=11$. These karyotype characteristics of $L$. apiculata are a common character within Myrtaceae.
\end{abstract}

Luma apiculata (DC.) Burret se distribuye latitudinalmente desde Valparaíso hasta Aisén y longitudinalmente cubre parte de la cordillera de los Andes, el Valle Central y la cordillera de Nahuelbuta (Rodríguez et al. 1983, Hoffmann 1997). Los estudios citogenéticos en L. apiculata son escasos y la información disponible sobre números cromosómicos es ambigua. En un primer conteo cromosómico realizado, se describió un número $\mathrm{n}=10(2 \mathrm{n}=20)$ (Titov de Tschischow 1956), mientras que en un conteo posterior se determinó un número $2 \mathrm{n}=22$ (Landrum 1981). Por otra parte, la morfología del cariotipo no ha sido descrita para el género Luma, el cual incluye dos especies endémicas, L. apiculata y L. chequen (Molina) A.Gray. Dentro de Myrtaceae, con alrededor de 3.800 especies a nivel mundial, se han realizado estudios cromosómicos en a lo menos 100 taxa y el $70 \%$ de ellos son diploides con un número cromosómico 22 . El porcentaje restante $(30 \%)$ corresponde a taxa poliploides que conservan también el número básico $\mathrm{x}=11$ (Atchinson 1947, Sanders et al. 1983, Dawson 2000, Mora et al. 2003, Costa \& Forni-Martins 2006, 2007). Es importante destacar, que los taxa chilenos de Myrtaceae están poco representados en los estudios citogenéticos actualmente disponibles y datos sobre números cromosómicos son conocidos solamente para seis especies pertenecientes a los géneros Luma, Myrceugenia y Ugni (Landrum 1981, Sanders et al. 1983). La morfología del cariotipo no ha sido descrita para esas especies.

Con el objetivo de incrementar los antecedentes citogenéticos existentes para el género Luma, en este trabajo se describe el cariotipo de L. apiculata.

Plantas y frutos maduros de L. apiculata fueron adquiridos en el vivero comercial Lusitania (catastrado por CTPF 2012), Región de La Araucanía, Provincia de Cautín, camino Temuco-Nueva Imperial, $\mathrm{km} \mathrm{28.} \mathrm{Las} \mathrm{plantas}$ son originarias de una población natural localizada en el fundo Rucamanque, Región de La Araucanía, Provincia de Cautín, noroeste de Temuco $\left(38^{\circ} 39^{\prime} \mathrm{S} ; 72^{\circ} 35^{\prime} \mathrm{W}\right)$. El material de referencia fue depositado en el herbario UCT (Universidad Católica de Temuco). En el laboratorio, un total de 100 semillas, provenientes de 10 plantas madres, fueron extraídas desde los frutos y germinadas a temperatura ambiente y fotoperíodo natural. Transcurridos 75 días, diez plántulas fueron utilizadas para el estudio cromosómico. Raíces de $5 \mathrm{~mm}$ de longitud fueron extraídas desde las plántulas y se trataron con 8-Hidroxiquinolina $2 \mathrm{mM}$, a temperatura ambiente durante $3 \mathrm{~h}$. Luego, las raíces fueron fijadas en etanol-ácido acético glacial (3:1 $\mathrm{v} / \mathrm{v})$ a $4^{\circ} \mathrm{C}$ por $24 \mathrm{~h}$ y posteriormente se tiñeron con la reacción de Feulgen. Los cromosomas fueron obtenidos por aplastado de meristemas radiculares y fotografiados con una cámara digital Olympus C-5050 conectada a un microscopio Olympus CX31. El conteo cromosómico se realizó sobre impresiones fotográficas ampliadas de diez metafases. La medición de brazos cortos y brazos largos de cada par cromosómico se realizó en cinco metafases utilizando el programa MicroMeasure 3.3 (Reeves 2001). Con esos valores de medición se determinó la longitud relativa de brazos cortos y brazos largos de cada cromosoma (expresada como porcentaje de la longitud total del juego haploide) y su morfología fue descrita siguiendo a Levan et al. (1964). Los cromosomas se representaron en un Idiograma y se ordenaron por longitud relativa decreciente. 
Adicionalmente, se estimó la longitud total del juego haploide (en $\mu \mathrm{m}$ ), el índice de asimetría TF\% de Huziwara (1962) y los índices de asimetría intracromosómica $A_{1}$ e intercromosómica $A_{2}$ de Romero-Zarco (1986).

Cromosomas metafásicos de L. apiculata y el Idiograma que representa la morfología del cariotipo se muestran en la Figuras 1 y 2. Las mediciones de los cromosomas se presentan en la Tabla I. Los especímenes de L. apiculata examinados en este trabajo muestran un número cromosómico $2 \mathrm{n}=$ 22, resultado que concuerda con el número documentado por Landrum (1981), pero que es distinto al $\mathrm{n}=10(2 \mathrm{n}=$ 20) descrito previamente por Titov de Tschischow (1956). El cariotipo de L. apiculata está constituido por 9 pares de cromosomas metacéntricos y 2 pares submetacéntricos (fórmula cariotípica haploide $9 \mathrm{~m}+2 \mathrm{sm}=11$ ). El par más largo tiene una longitud de $2,2 \mu \mathrm{m}$ y excede en más del doble (2,7 veces) el tamaño del par más corto que mide $0,8 \mu \mathrm{m}$. El cariotipo es simétrico, con un valor de $\mathrm{TF} \%=42,07 \mathrm{y}$ valores de $A_{1}=0,26 \pm 0,02$ y $A_{2}=0,25 \pm 0,04$. La longitud total del juego haploide es 16,4 $\pm 0,04 \mu \mathrm{m}$. Todos estos caracteres cuantitativos descritos en el cariotipo de L. apiculata, muestran valores que están dentro de los rangos que se han documentado para otros taxa de la familia (rango de $\mathrm{A}_{1}=0,19$ 0,42 y rango de $\mathrm{A}_{2}=0,14-0,35$; rango de $\mathrm{TF} \%=35,84-45,37$ ) (Costa \& Forni-Martins 2007). Los análisis cuantitativos de los cariotipos hasta ahora estudiados en Myrtaceae, basados en el índice centromérico e índices de asimetría, han permitido caracterizar especies, pero no son resolutivos para diferenciar a nivel de géneros o subtribus (Costa \& ForniMartins 2007). De esta forma, es indispensable la aplicación de técnicas de bandeo (i. e., C, Ag-NOR, FISH), lo cual permitiría incrementar el nivel de resolución para identificar cromosomas marcadores, tanto en el género Luma como en otros géneros de Myrtaceae, y aportaría también un nuevo conjunto de datos genómicos para el estudio (o re-estudio) de relaciones filogenéticas y taxonómicas dentro de la familia (Jara-Seguel \& Urrutia 2012).

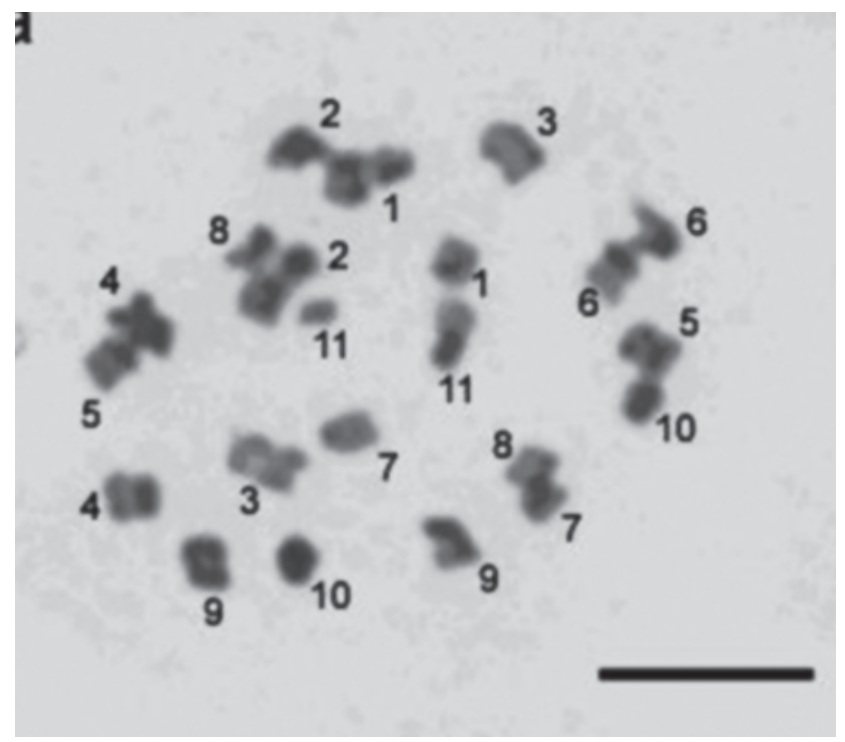

Figura 1. Metafase mitótica de Luma apiculata, $2 \mathrm{n}=22$. Barra $=5 \mu \mathrm{m}$.

Figure 1. Mitotic metaphase of Luma apiculata, $2 \mathrm{n}=22$. Bar $=$ $5 \mu \mathrm{m}$.

TABLA I. Características cuantitativas del cariotipo de Luma apiculata. BC, longitud relativa del brazo corto; BL, longitud relativa del brazo largo; LT, longitud relativa total; TC, tamaño cromosómico absoluto; IC, índice centromérico; FC, forma cromosómica; $\mathrm{X} \pm \mathrm{DE}, \mathrm{media} \pm$ desviación estándar.

TABLE I. Quantitative characteristics of the karyotype of Luma apiculata. BC, relative length of the short arm; BL, relative length of the long arm; LT, total relative length; TC, absolute chromosome size; IC, centromeric index; FC, chromosome shape; $\pm \pm D E$, mean \pm standard deviation.

\begin{tabular}{|c|c|c|c|c|c|c|}
\hline PAR CROMOSÓMICO & $\begin{array}{l}\mathrm{BC}(\%) \\
\mathrm{X} \pm \mathrm{DE}\end{array}$ & $\begin{array}{l}\mathrm{BL}(\%) \\
\mathrm{X} \pm \mathrm{DE}\end{array}$ & $\begin{array}{l}\mathrm{LT}(\%) \\
\mathrm{X} \pm \mathrm{DE}\end{array}$ & $\begin{array}{l}\mathrm{TC}(\mu \mathrm{m}) \\
\mathrm{X} \pm \mathrm{DE}\end{array}$ & $\begin{array}{c}\mathrm{IC} \\
\mathrm{X} \pm \mathrm{DE}\end{array}$ & FC \\
\hline 1 & $6,1 \pm 0,04$ & $7,6 \pm 0,03$ & $13,7 \pm 0,05$ & $2,2 \pm 0,03$ & $0,44 \pm 0,03$ & $\mathrm{~m}$ \\
\hline 2 & $6,1 \pm 0,05$ & $6,1 \pm 0,02$ & $12,2 \pm 0,03$ & $2,0 \pm 0,05$ & $0,50 \pm 0,04$ & $\mathrm{~m}$ \\
\hline 3 & $4,6 \pm 0,03$ & $6,1 \pm 0,04$ & $10,7 \pm 0,04$ & $1,7 \pm 0,02$ & $0,43 \pm 0,02$ & $\mathrm{~m}$ \\
\hline 4 & $4,6 \pm 0,02$ & $4,6 \pm 0,06$ & $9,1 \pm 0,03$ & $1,5 \pm 0,04$ & $0,50 \pm 0,04$ & $\mathrm{~m}$ \\
\hline 5 & $4,6 \pm 0,04$ & $4,6 \pm 0,03$ & $9,1 \pm 0,02$ & $1,5 \pm 0,03$ & $0,50 \pm 0,02$ & $\mathrm{~m}$ \\
\hline 6 & $2,0 \pm 0,06$ & $6,1 \pm 0,02$ & $8,1 \pm 0,04$ & $1,3 \pm 0,04$ & $0,25 \pm 0,06$ & sm \\
\hline 7 & $2,0 \pm 0,05$ & $6,1 \pm 0,04$ & $8,1 \pm 0,03$ & $1,3 \pm 0,02$ & $0,25 \pm 0,05$ & $\mathrm{sm}$ \\
\hline 8 & $3,0 \pm 0,04$ & $4,6 \pm 0,06$ & $7,6 \pm 0,04$ & $1,2 \pm 0,04$ & $0,40 \pm 0,03$ & $\mathrm{~m}$ \\
\hline 9 & $3,0 \pm 0,03$ & $4,6 \pm 0,05$ & $7,6 \pm 0,02$ & $1,2 \pm 0,05$ & $0,40 \pm 0,04$ & $\mathrm{~m}$ \\
\hline 10 & $3,0 \pm 0,05$ & $4,6 \pm 0,04$ & $7,6 \pm 0,04$ & $1,2 \pm 0,02$ & $0,40 \pm 0,05$ & $\mathrm{~m}$ \\
\hline 11 & $3,0 \pm 0,06$ & $3,0 \pm 0,03$ & $6,1 \pm 0,06$ & $0,8 \pm 0,04$ & $0,50 \pm 0,03$ & $\mathrm{~m}$ \\
\hline
\end{tabular}




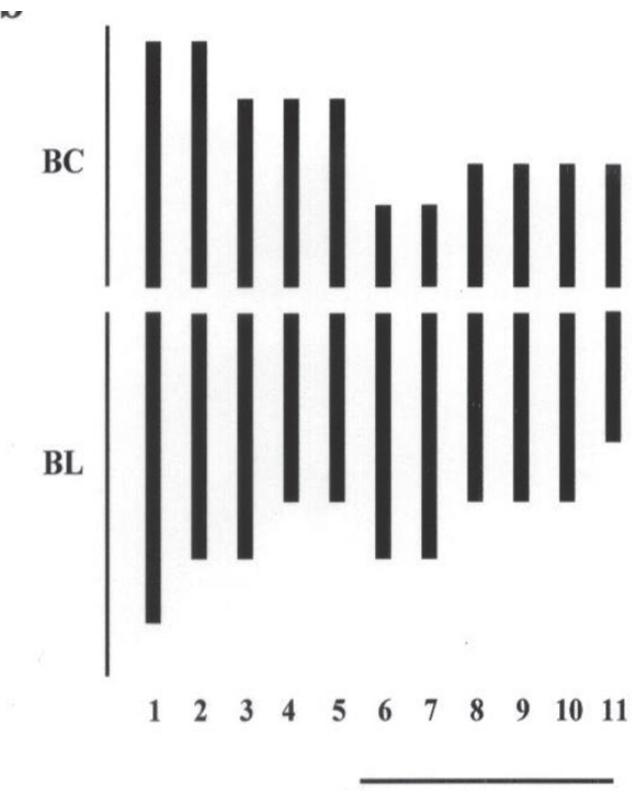

FIgURA 2. Idiograma de Luma apiculata, $2 \mathrm{n}=22$. Los cromosomas 6 y 7 son submetacéntricos y los restantes son metacéntricos. BC, brazo corto; BL, brazo largo. Barra $=1 \mu \mathrm{m}$.

FIgURE 2. Idiogram of Luma apiculata, $2 \mathrm{n}=22$. Chromosomes 6 and 7 are submetacentric, and the remaining chromosomes are metacentric. BC, short arm; BL, long arm. Bar $=1 \mu \mathrm{m}$.

\section{AGRADECIMIENTOS}

A Mario Romero por la herborización del material de $L$. apiculata utilizado en este estudio.

\section{BIBLIOGRAFÍA}

Atchinson, E. 1947. Chromosome numbers in the Myrtaceae. American Journal of Botany 34: 159-164.

Costa, I. \& E. Forni-Martins. 2006. Chromosome studies in Brazilian species of Campomanesia Ruiz et Pavon and
Psidium L. (Myrtaceae Juss.). Caryologia 59: 7-13.

Costa, I. \& E. Forni-Martins. 2007. Karyotype analysis in South American species of Myrtaceae. Botanical Journal of the Linnean Society 155: 571-580.

Dawson, M. 2000. Index of chromosome numbers of indigenous New Zealand Spermatophytes. New Zealand Journal of Botany 38: 47-150.

Hoffmann, A. 1997. Flora Silvestre de Chile, Zona Araucana. Ediciones Fundación Claudio Gay, Santiago, Chile.

Huziwara, Y. 1962. Karyotype analysis in some genera of Compositae. VIII. Further studies on the chromosomes of Aster. American Journal of Botany 49: 116-119.

CTPF. 2012. Centro Tecnológico de la Planta Forestal: Grupo de investigación en producción y establecimiento de plantas. Instituto Forestal (INFOR). URL: http://www.ctpf.cl/ produccion-viveros-forestales.html. (Acceso: $15 \mathrm{de}$ noviembre de 2012).

Jara-Seguel, P. \& J. Urrutia. 2012. Cytogenetic of Chilean Angiosperms: Advances and prospect. Revista Chilena de Historia Natural 85:1-12.

LANDRUM, L.R. 1981. A monograph of the genus Myrceugenia (Myrtaceae). Flora Neotropica Monographs 29: 1-137.

Levan, A., K. Fredga \& A. Sandberg. 1964. Nomenclature for centromeric position on chromosomes. Hereditas 52: 201219.

Mora, F., C. Palma-Rojas \& P. Jara-Seguel. 2003. Comparación del cariotipo de Eucalyptus globulus y Eucalyptus cladocalyx (Myrtaceae). Agricultura Técnica (Chile) 65(1): 20-25.

ReEves, A. 2001. MicroMeasure: A new computer program for the collection and analysis for the cytogenetic data. Genome 44: 239-443.

Rodríguez, R., O. Matthei \& M. Quezada. 1983. Flora Arbórea de Chile. Editorial de la Universidad de Concepción, Concepción, Chile. 408 pp.

Romero-Zarco, C. 1986. A new method for estimating karyotype asymmetry. Taxon 35: 526-530.

Sanders, R., T. Stuessy \& R. Rodríguez 1983. Chromosome numbers from the Flora of the Juan Fernandez Islands. American Journal of Botany 70(6): 799-810.

Titov de Tschischow, N. 1956. Número de cromosomas de algunas plantas chilenas. Boletín de la Sociedad de Biología de Concepción, Tomo III: 145-147.

Recibido: 07.01 .13

Aceptado: 27.05.13 